\title{
The Anatomy of the Cerebral Cortex
}

\author{
Pradip Chauhan ${ }^{1}$ - Ashish Rathawa ${ }^{2}$ - Kinjal Jethwa ${ }^{3}$ - Simmi Mehra ${ }^{1}$ \\ ${ }^{1}$ Department of Anatomy, All India Institute of Medical Sciences, Rajkot, Gujarat, India; \\ ${ }^{2}$ Department of Anatomy, GMERS Medical College, Junagadh, Gujarat, India; \\ ${ }^{3}$ Department of Anatomy, SKBS Medical College and Research Institute, Sumandeep \\ Vidhyapeeth, Baroda, Gujarat, India
}

Author for correspondence: Pradip Chauhan, Department of Anatomy, All India Institute of Medical Sciences, Rajkot, Gujarat, India. Email: prajjawalitresearch@gmail.com

Cite this chapter as: Chauhan P, Rathawa A, Jethawa K, Mehra S. The Anatomy of the Cerebral Cortex. In: Pluta R, editor. Cerebral Ischemia. Brisbane (AU): Exon Publications; 2021. Online first Sep 02.

Doi: https://doi.org/10.36255/exonpublications.cerebralischemia.2021.cerebralcortex

\begin{abstract}
The cerebral hemisphere consists of five lobes: frontal, parietal, temporal, occipital, and limbic lobe. Each cerebral hemisphere shows superomedial, inferior, and medial surfaces separated by superomedial, inferomedial, and inferolateral borders. The superolateral surface shows the central sulcus that separates the pre-central and post-central gyri. The parietal lobe is divided by the interparietal sulcus into supra-parietal and infra-parietal lobes. The occipital lobe contains the primary visual area surrounded by peristriate and parastriate areas. The temporal lobe is divided into superior, middle, and inferior temporal gyri. The superior surface of the superior temporal gyrus is occupied by the primary and secondary speech areas. The medial surface shows C-shaped corpus callosum, cingulate gyrus, medial frontal gyrus, cuneus, precuneus, cingulate sulcus and paracentral lobule. The orbital part of the inferior surface shows H-shaped orbital sulcus, olfactory sulcus, and olfactory gyrus. Broca's motor speech area is present in the dominant hemisphere at the inferior frontal gyrus. Wernicke's speech area is present in supramarginal and angular gyri. The cerebral hemisphere is mainly supplied by anterior, middle, and posterior cerebral arteries. Understanding the anatomy of the cerebral cortex is critical to recognize the site of lesion in cerebral ischemia.
\end{abstract}

In: Cerebral Ischemia. Pluta R (Editor). Exon Publications, Brisbane, Australia. ISBN: 978-0-6450017-9-2; Doi: https://doi.org/10.36255/exonpublications. cerebralischemia.2021

Copyright: The Authors.

License: This open access article is licenced under Creative Commons Attribution-NonCommercial 4.0 International (CC BY-NC 4.0) https://creativecommons.org/licenses/by-nc/4.0/ 
Keywords: cerebral cortex; functional areas; insula; primary motor area; primary sensory area

\section{INTRODUCTION}

The cerebrum is part of the central nervous system situated in the cranial cavity and connected to the cerebellum through the brainstem (1). The volume of the cerebrum is $1,060.0 \pm 94.6$ and $1,008.9 \pm 104.3 \mathrm{~cm}^{3}$ in males and females, respectively, which is $87 \%$ of total brain volume (1). Median longitudinal fissure separates the cerebrum into two cerebral hemispheres attached with each other through a bundle of white fibers-the corpus callosum (2). Each cerebral hemisphere has a cortical grey matter, a white matter beneath the grey matter, and a collection of subcortical neuronal masses within the white matter basal ganglia $(1,2)$. Each cerebral hemisphere has a cavity which is called the lateral ventricle; the lateral ventricles communicate with the third ventricle through the anterior interventricular foramen $(1,2)$. The ventricles contain cerebrospinal fluid, a nutrient material for the brain tissue (2).

\section{EXTERNAL FEATURES}

Each cerebral hemisphere has three borders, three surfaces, three poles, and six lobes. External surfaces of the cerebrum show elevations known as gyri and depressions known as sulci (2-5).

\section{Poles}

Each cerebral hemisphere has three poles: frontal, temporal, and occipital pole (2). The anterior most end of the cerebral hemisphere is known as the frontal pole that correlates with the superciliary arch (2). The posterior most end of the cerebral hemisphere is known as the occipital pole while the temporal pole is the anterior most end of the temporal lobe (2-5). The temporal pole occupies the anterior part of the middle cranial fossa (2-5).

\section{Surfaces}

Each cerebral hemisphere has three surfaces (Figure 1): superolateral surface, medial surface, and inferior surface (2-5). The superolateral surface is convex and faces towards the cranial vault; it is the most extensive of all surfaces (2-5). The medial surface is flat and faces towards the medial surface of another hemisphere. The space between medial surfaces of two hemispheres is known as longitudinal cerebral fissure which is occupied by falx cerebri. C-shaped cut part of the corpus callosum is the characteristic of the medial surface (2-5). Inferior surface faces towards the base of the cranial cavity. The stem of the lateral sulcus divides the inferior surface into orbital and tentorial parts. The anterior part of the inferior 
A

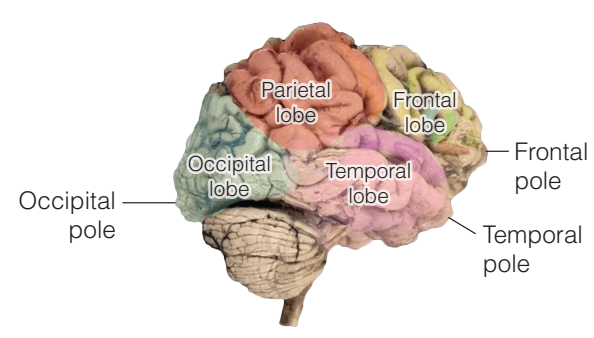

C

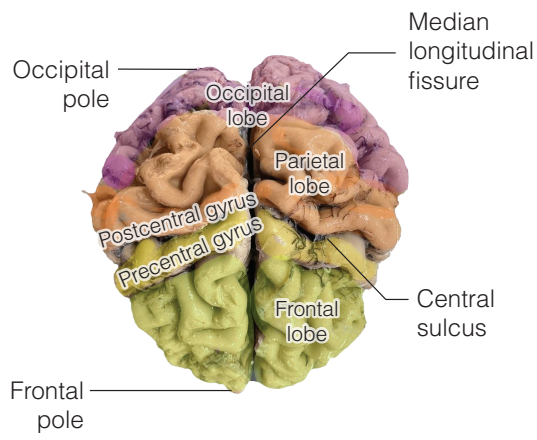

\section{B}

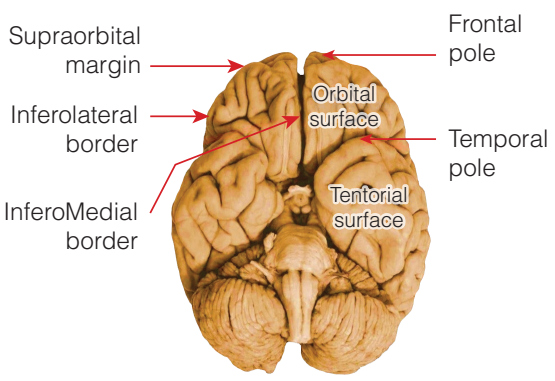

D

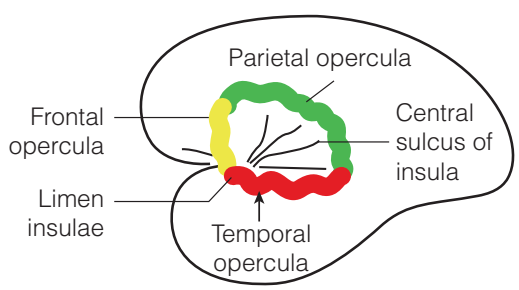

Figure 1. Borders, surfaces, poles, and lobes of the cerebral hemisphere. A, Superolateral surface. B, Inferior surface. C, Superior surface. D, Insular lobe.

surface lies over the orbital plate and anterior cranial fossa and it is known as the orbital part. The posterior large part rests over the tentorium cerebelli and is known as the tentorial part.

\section{Borders}

The superomedial border extends from the frontal pole to the occipital pole (Figure 1); it separates the superomedial surface from the medial surface $(4,5)$. The inferolateral margin of cerebral hemisphere separates the superolateral surface of the cerebral hemisphere from the inferior surface. The part of the inferolateral border anterior to the stem of the lateral sulcus is named the superciliary border (3-5). The inferomedial border separates the medial surface from the inferior surface. It is interrupted by the brainstem and the diencephalon in the anterior medial orbital border and posterior medial occipital border (3-5).

\section{SULCI AND GYRI}

To include the extensive functional area, the cerebrum is folded into various convolutions known as gyri (Figure 2). The fissures between convolutions are known as sulci. The mean cortical surface area of the adult human brain is $2000 \mathrm{~cm}^{2}$, of 
A

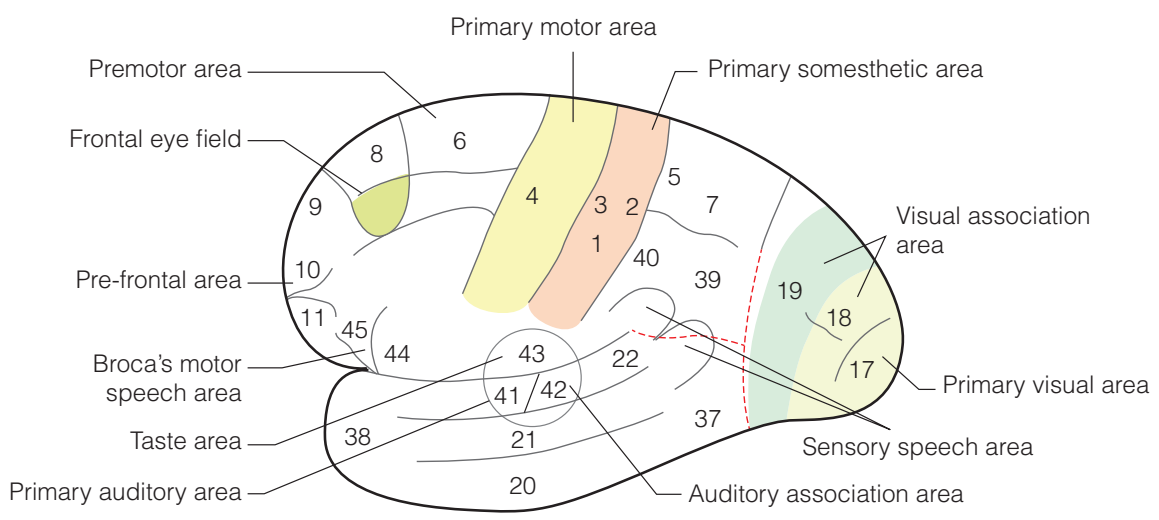

B

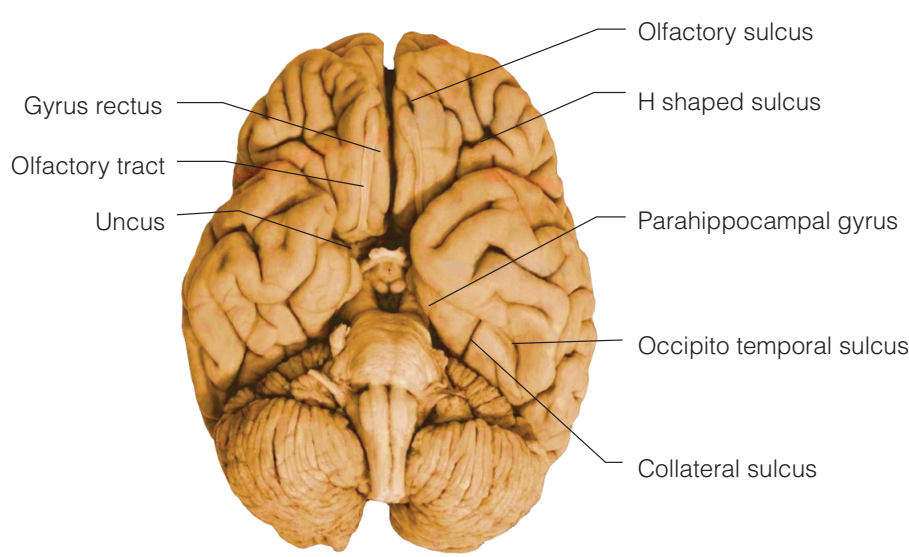

C

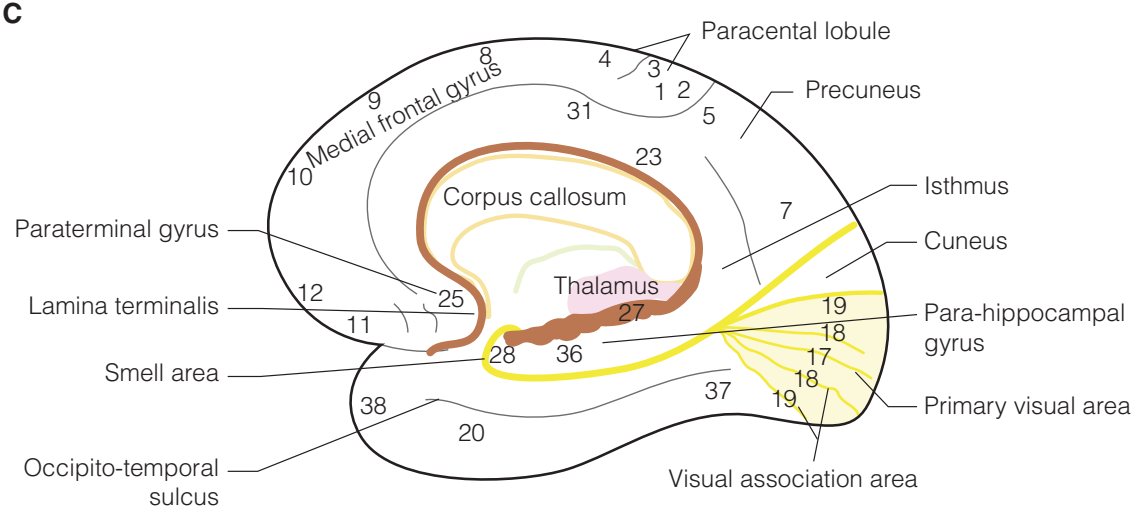

Figure 2. Sulci, gyri, and functional areas. A, Superolateral surface. B, Inferior surface.

C, Medial surface. 
which two-third is hidden in the sulci (4-5). The brain with convolutions is termed gyrencephalic and that without convolutions is known as lissencephalic (4). Depth of sulci varies from slight grooving to deep enough to invaginate the lateral ventricle. The core of gyri contains white matter while its outer area contains grey matter (3-6). On the basis of morphology, sulci can be classified into various types (3-6).

(i) Large primary sulci: central, precentral, and postcentral sulci are examples of large primary sulci.

(ii) Short primary sulci: rhinal, olfactory, occipital, and lateral sulci are examples of short primary sulci.

(iii) Short sulci composed of several branches: subparietal and orbital sulci are examples of short sulci with several branches.

(iv) Short free supplementary sulci: lunate and medial frontal sulci are examples of short, free supplementary sulci. Some sulci are composed of side branches that connect 2 neighboring parallel sulci.

The lateral sulcus of Sylvius, Central sulcus of Rolando, Calcarine sulcus, and Parieto-occipital sulcus are the four main sulci that are constant and divide the cerebral hemisphere into five lobes $(5,6)$. The lateral Sulcus of Sylvius has a stem and three branches; the stem extends from the inferior surface of the cerebral hemisphere as a deep cleft just anterior to the anterior perforated substance to the superolateral surface of the cerebral hemisphere. It delimitates the orbital surface and temporal lobe $(5,6)$. The stem divides into three branches (anterior horizontal, anterior ascending, and posterior) on the lateral surface of the cerebrum. $(5,6)$ The central sulcus extends from a point $1 \mathrm{~cm}$ behind the midpoint between the frontal and occipital pole on the superomedial border to just above the lateral sulcus (4). On the medial surface of the cerebrum, it is bounded by the paracentral lobule $(5,6)$. The central sulcus divides the primary motor area anterior to it from the primary sensory area posterior to it (4-6). The calcarine sulcus is present on the medial surface of the cerebral hemisphere (4). It extends from little below the splenium (posterior end of corpus callosum) to the occipital pole (4-6). Parieto-occipital sulcus starts as a branch from the midpoint of the calcarine sulcus on the medial surface of the hemisphere and turns to the occipital pole, which extends on the superolateral surface (4-6).

\section{LOBES}

The cerebral hemisphere is divided into five lobes (Figures 1A-C): frontal lobe, parietal lobe, temporal lobe, occipital lobe, and insula (2, 4-6). Two imaginary lines are drawn on the cerebral hemisphere. The first is a vertical line from the parieto-occipital sulcus to the pre-occipital notch (2). The second imaginary line extends from the posterior ramus of the lateral sulcus that meets the first vertical line (2). Two imaginary lines and three main sulci (central, lateral, and pariteooccipital sulci) divide the cerebral hemisphere into five lobes $(2,4-6)$. The frontal lobe lies anterior to the central sulcus and above the lateral sulcus $(2,4-6)$. The parietal lobe is delimited anteriorly by the central sulcus, posteriorly by the vertical imaginary line (joining the parieto-occipital sulcus and pre-occipital notch) 
and inferiorly by the horizontal imaginary line (extending from the posterior ramus of lateral sulcus to the first imaginary vertical line) (4-6).

The temporal lobe is limited above by the posterior ramus of the lateral sulcus and second imaginary line and inferiorly by the inferolateral border (4-7). The occipital lobe lies behind the first imaginary vertical line joining the parietooccipital sulcus and pre-occipital notch (4-9). Insula (Central lobe) or the island of Reil is a hidden portion of the cerebral cortex on the floor of the lateral sulcus (4). Insula is covered by overgrown cortical areas of frontal, parietal and temporal lobes known as a frontal operculum, parietal operculum, and temporal operculum respectively (7). The apex of the insula is termed as limen Insula that is continuous with the anterior perforated substance (8). The circular sulcus covers the Insula all around except at the apex $(4,7,9)$. Sulcus centralis divides the insula into anterior and posterior parts. The anterior part contains 3-4 short gyri known as gyri brevia and the posterior part contains 1-2 long gyri known as gyri longa $(4,10)$. Functions of Insula are as follows $(4,10)$ : (i) awareness of the present movement; (ii) autonomic function control by sympathetic and parasympathetic activity; (iii) integration with the limbic system, it is involved in love, emotion, and consciousness; and (iv) coordination of articulatory speech movements.

\section{Sulci and gyri present on the superolateral surface}

The central sulcus is between the frontal lobe and parietal lobe (2-5). Gyrus present anterior to the central sulcus is the precentral gyrus. The precentral gyrus is limited anteriorly by the precentral sulcus (Figure1A, 1C, 2A) $(1,5-7,10)$. Two horizontal sulci run parallel anterior to the precentral sulci which divide the frontal lobe into superior, middle, and inferior frontal gyri $(5-7,10)$. The inferior frontal gyrus is divided by the anterior horizontal and anterior ascending rami of the lateral sulcus into three parts (5). The part below the anterior horizontal ramus is known as pars orbitalis, the part between the anterior horizontal and anterior ascending is known as pars triangularis, and the part posterior to the ascending ramus is known as pars opercularis $(5-7,10)$.

Gyrus present posterior to the central sulcus is known as postcentral gyrus. The postcentral gyrus is limited posteriorly by the postcentral sulcus (5). The parietal lobe is further divided by the intraparietal sulcus into superior and inferior parietal lobule (5-9). The inferior parietal lobule is further divided into three parts. The part surrounding the upturned end of the posterior ramus is known as the supramarginal gyrus, the part surrounding the superior temporal sulcus is known as the angular gyrus, and the part surrounding the posterior end of the inferior temporal sulcus known as pars temporal-occipitalis (6-9). Two parallel sulci, superior and inferior temporal sulci, present on the temporal lobe divide the temporal lobe into three gyri: superior, middle, and inferior temporal gyri $(7,8)$. Two transverse temporal sulci are present on the superior surface of the superior temporal gyrus (8). The anterior transverse temporal gyrus (Heschl's gyrus) shows the primary auditory area of the cortex (5-9).

\section{Sulci and gyri present on the medial surface}

The flat medial surface of each cerebral hemisphere presents the C-shaped bundle of white fibers known as the Corpus callosum (Figure 2C) (5). It presents from 
anterior to posterior the rostrum, genu, body, and splenium (5). Genu is the sharp anterior bend of the corpus callosum that is present $4 \mathrm{~cm}$ posterior to the frontal pole (6). Splenium is a comparatively larger part positioned $6 \mathrm{~cm}$ anterior to the occipital pole (5). The rostrum continues downward as lamina terminalis that makes the anterior border of the third ventricle $(5,9)$. Lamina terminalis is a thin sheet of grey matter which extends from the rostrum to the upper surface of the optic chiasma; in addition, it presents the cranial end of the primitive neural tube $(6,9)$. The convex superior surface of the corpus callosum forms the floor of the median longitudinal fissure; the surface is covered by a thin sheet of grey matter known as indusium griseum $(5,9)$. The indusium griseum is continuous as a para-terminal gyrus anteriorly $(5,9)$. Posterior continuation of the indusium griseum forms the gyrus fascioliasis that covers the splenium, then continues as the dentate gyrus of the temporal lobe $(5,6,9)$.

A thin sheet of grey matter anterior to the lamina terminalis is known as paraterminal gyrus, which is continuous with the diagonal band of Broca and the medial olfactory stria on the inferior surface of the cerebrum (5, 7-9). The parolfactory gyrus (Subcallosal area) lies anterior to the paraterminal gyrus which is bounded by the anterior and posterior parolfactory sulci (5, 7-9).

A sulcus between the corpus callosum and the cingulate gyrus is known as the callosal sulcus. The cingulate sulcus surrounds the cingulate gyrus, it runs parallel to the curvature of the corpus callosum. Anteriorly it ends below the genu of the corpus callosum while the posterior end of the cingulate sulcus upturns and reaches the superomedial border $4 \mathrm{~cm}$ behind the central sulcus and makes the posterior boundary of the paracentral lobule. The medial surface of the cerebral hemisphere is divided into inner and outer zones by the cingulate sulcus. The inner zone is named as cingulate gyrus that is continuous as para-hippocampal gyrus on the tentorial surface through the isthmus $(5,7)$.

A vertical sulcus further subdivides the outer zone; anterior to the vertical sulcus is known as the medial frontal gyrus and posterior to the vertical sulcus is known as the paracentral lobule (7). The paracentral lobule can be divided into two parts by the commencement of the central sulcus (7-10). The part anterior to the central sulcus is continuous with the precentral gyrus and regulates the movement of the contralateral leg and foot, voluntary control of the defecation, and micturition $(9,10)$. The part posterior to the central sulcus continues with the post-central gyrus and receives somesthetic sensations from the contralateral leg and foot; it also receives a sense of distension from the bladder and rectum $(5,7-10)$.

The parieto-occipital and calcarine sulci are present behind the corpus callosum (Figure 2C). Parieto-occipital sulcus extends from the superomedial border, passes downward and forward to fuse with the anterior part of the calcarine sulcus. The area anterior to the parieto-occipital sulcus and posterior to the upturned end of the cingulate sulcus is known as precuneus. The precuneus is bounded inferiorly by a disrupted sub-parietal (suprasplenial) sulcus (5, 7-10).

The calcarine sulcus starts just below the splenium of the corpus callosum and extends up to the occipital pole, it receives parieto-occipital sulcus in the middle part. The triangular gyrus between the parieto-occipital and calcarine sulcus is known as the cuneus. The posterior end of the calcarine sulcus meets with the lunate sulcus. The posterior part of the calcarine sulcus is an example of the axial sulcus as the visual cortex grows along with it. The anterior end of the calcarine 
sulcus presents a complete sulcus because it invaginates the posterior horn of the lateral ventricle and forms calcarine avis $(5,7-10)$. The cortical area below the calcarine sulcus is known as the lingual gyrus (8). Anteriorly the lingual gyrus continues as the para-hippocampal gyrus in the temporal lobe $(5,8,10)$.

\section{Sulci and gyri present on the inferior surface}

The stem of the lateral sulcus divides the inferior surface into the orbital surface anteriorly and the tentorial surface posteriorly (Figure 1B,2B) (10). The anterior perforated substance is bounded in front by the olfactory trigone and is located at the commencement of the lateral sulcus (10). The anterior perforated substance is perforated by the central branches of the middle cerebral artery $(5,7,10)$. The olfactory trigone is bounded by the divergence of medial and lateral olfactory stria anteriorly, medially by the optic chiasma, and posterolaterally by the uncus of the temporal lobe $(5,7,10,11)$.

The orbital surface presents olfactory sulcus near the inferomedial border (5). The olfactory sulcus lodges the olfactory bulb and its tract (9-12). The olfactory tract diverges into medial and lateral olfactory stria (9-12). The cortical area medial to the olfactory sulcus is known as the gyrus rectus (9-12). The area medial to the olfactory sulcus is further subdivided by the $\mathrm{H}$ shaped sulcus into four different gyri: the anterior, posterior, medial, and lateral olfactory gyri $(5,9-12)$.

The anterior part of the tentorial surface is lodged in the middle cranial fossa and the posterior part rests on the tentorial cerebelli $(5,10-12)$. The occipitotemporal and collateral sulci divide the tentorial surface into lateral occipitotemporal gyrus, medial occipitotemporal gyrus, and parahippocampal gyrus $(5,9-12)$.

The collateral sulcus produces collateral eminence into the inferior horn of the lateral ventricle $(5,8-12)$. Anteriorly the collateral sulcus is continuous as the rhinal sulcus which makes the lateral boundary of the uncus (8-12). The uncus acts as the primary receptive area of the olfaction (8-12). The uncus continues posteriorly as the parahippocampal gyrus. The dentate gyrus is a crenated strip present superomedially the parahippocampal gyrus separated by hippocampal sulcus (8-12). Posterior continuation of the dentate gyrus is known as gyrus fasciolaris, anteriorly the dentate gyrus bends medially as a band of Giocomi $(5,9-12)$.

The dentate gyrus is covered by the free margin of the fimbria hippocampi; the sulcus between the dentate gyrus and fimbria hippocampi is known as the fimbriodentate sulcus $(5,9)$. Choroid fissure is present above the fimbria through which the choroid plexus enters into the inferior horn of the lateral ventricle $(5,9-12)$.

\section{HISTOLOGICAL STRUCTURE OF THE CEREBRAL CORTEX}

Neurons present in the cerebral cortex are pyramidal cells, stellate cells, horizontal cells of Cajal, Fusiform cells and cells of Martinotti (13-16). The pyramidal cells have apices directed towards the external surface. Axons arise from the center of the base of pyramidal cells while dendrites originate from angles of the pyramidal 
cells (13-17). The axons of the pyramidal cells enter the white matter as projection fibers. The pyramidal cells are further classified as small, medium, and large size (14-17). The stellate/granule cells are around $8 \mu \mathrm{m}$ in diameter and star-shaped in size (14-17). Stellate cells look like nizzle-stained granules so-known as Granule cells. Granule cells have small axons and multiple dendrites. Some areas of the cerebral cortex show clouding (collection) of the granule cells; such areas are known as Koniocortex (Konio = Cloud) $(15-17)$. The horizontal cells of Cajal are fusiform in shape and oriented horizontally in the most superficial layer of the cortex. The fusiform cells are also fusiform in shape, but they are oriented vertically and observed in the deep layer of the cortex (15-17). The cells of Martinotti are small multipolar cells and found in all layers of the cerebral cortex (13-17).

\section{Laminar organization in the cerebral cortex}

Microscopically, the following horizontal organizations of cellular layers (Laminae) are observed $(5,13-17)$ :

(i) Molecular (Plexiform) layer I: consists of apical dendritic tufts of the pyramidal neurons, horizontally organized axons, Cajal-Retzius cells and glial cells.

(ii) External granular layer II: consist of mainly granular cells and small pyramidal cells.

(iii) External pyramidal layer III: consists of small and medium-sized pyramidal cells. Layer III can be divided into most superficial IIIa, intermediate layer IIIb and deep layer IIIc. Horizontally running myelinated fibers makes the band of Baillarger stripe that can be appreciated between layer IIIc and IV in some isocortical regions.

(iv) Internal granular layer IV: contains small round granular cells (spiny stellate cells, spiny interneurons) and small pyramidal cells. Layer IV shows the band of Baillarger on the outer side conspicuous in the visual cortex; the band is named as the stripe of Gennari or of Vicq d'Azyr.

(v) Internal pyramidal layer V: contains the largest pyramidal cells. Axons of the pyramidal cells leave the cortex and reach subcortical areas. Layer V is subdivided into Va, Vb and Vc sublayers. The inner band of Baillarger is observed in layer $\mathrm{V}$ in myelin-stained sections.

(vi) Multiform (Polymorphous) layer VI: Excitatory cells like spiny stellate cells, pyramidal cells, inverted pyramidal neurons, bipolar/fusiform cells, and odd-shaped cells are present in layer VI. It also contains inhibitory interneurons. This layer is also divided into sublayers VIa and VIb.

The homotypical cortex is the part of the cerebral cortex where all six layers are well defined. The heterotypic cortex is the part of the cerebral cortex where all six layers are not well defined. It is further subdivided into the granular and agranular cortex. In the granular cortex, granular layers are well defined while pyramidal layers are poorly defined; granular cortex is mostly seen in sensory areas. In the agranular cortex, granular layers are poorly defined while pyramidal layers are well defined. The agranular cortex is mostly seen in motor areas. 


\section{PHYLOGENETIC CLASSIFICATION OF THE CEREBRAL CORTEX}

Phylogenetically, the cerebral cortex is classified as an isocortex, which is homogenetic, and allocortex, which is heterogenetic (14-17). Allocortex can be further classified as archicortex and paleocortex. Isocortex, or the neocortex, developed late during brain evolution; it coincides with the appearance of the first mammalian species. All areas of the isocortex are comprised of all six histological layers. The adult isocortex is either eulaminate (homotypical) or koniocortical (heterotopic/agranular). Eulaminate isocortex is found in most of the prefrontal, parietal, temporal, and occipital cortices. The heterotypical koniocortical cortex is characterized by the presence of layer IV granule cells in more superficial layers; examples are primary somatosensory, visual, and auditory areas. Paleocortex is the intermediate in phylogenetic origin. The human cingulated gyrus is an example of the paleocortex. Archicortex is the oldest in phylogenetic origin; structurally it is comprised of only three layers. The agranular cortex contains more pyramidal cells in layer IV than layers III and layer V; examples of agranular areas are the motor cortex, prefrontal area BA8, posterior part of Broca's language region (BA4).

\section{FUNCTIONAL AREAS OF THE CEREBRAL CORTEX}

Brodmann divided the cerebral cortex into 47 areas (Brodmann's areas, BA) according to structure and functions (Figure 2A, 2B, 2C) (18). Further, the cerebral cortex can be divided into various cytoarchitectonic areas on the basis of Von Economo and Koskinas classification and recent studies $(18,19)$. The cerebral cortex contains three types of functional areas: motor areas, sensory areas, and association areas. Motor areas control motor activities, while sensory areas receive sensory information through afferent fibers via thalamic nuclei. Association areas are important for associative, cognitive, and integrative functions $(2,11,18,19)$.

The primary motor area (BA4) is present in the anterior wall of the central sulcus, it contains large pyramidal cells which send projection fibers in the form of corticospinal and cortico-nuclear fibers (2). This area shows the lowest threshold for eliciting the contralateral muscle contraction (5). The topographically organized map of the opposite half of the body is presented in an inverted manner in the area which is called a motor homunculus (8). The head is represented most laterally and inferiorly, the hand is represented in the central part while the leg and foot are represented in the medial surface of the paracentral lobule (11). In the motor homunculus, body areas are represented in proportion to the precision and skill of the movement performed by the body region rather than their physical size; face and hand muscles occupy large areas while the thigh muscle occupies a small area $(18,19)$.

The Premotor area (BA6) is present anterior to the primary motor area in the posterior part of the superior, middle, and inferior frontal gyri, and extends on the medial surface of the hemisphere (11). It lacks the giant pyramidal cells (Cells of Betz) and is the main cortical origin of the extrapyramidal tracts (18). The Premotor area receives sensory input and stores past data. On the basis of 
previous experiences, it programs intended motor activities, so it has a crucial role in voluntary skilled movements $(2,11,18,19)$.

The supplementary motor area (SMA, MsII) is on the medial surface of the hemisphere just in front of the paracentral lobule (5). The Presupplementary motor area (Pre SMA) lies anterior to the supplementary motor area on the medial surface of the cortex (11). The body is represented in the supplementary motor area from anterior to posterior in craniocaudal order (18). The supplementary motor area controls the movements in complex tasks that require retrieval of memory and temporal organization of sequential movements $(5,11,18,19)$.

The frontal eye field (FEF, BA8) is located near the junction of the middle frontal and precentral sulci (2). The frontal eye field is responsible for voluntary scanning movements of the eye (Conjugate eye movement) and moves eyes to the opposite side (5). The frontal eye field is connected to the superior colliculus, substantia nigra and the dentate nucleus of the cerebellum through the thalamocortical pathway (18-20). It receives input from the parietal lobe, temporal lobe, occipital lobe visual area and middle temporal area $(2,5,18-21)$.

Broca's motor speech area 44 (BA44) is located at pars triangularis, and Broca's motor speech area 45 (BA45) is located at pars opercularis in the inferior frontal gyrus of the dominant cerebral hemisphere (18-20). This area receives input from the posterior and middle superior temporal gyri including auditory association areas; it also receives fibers from the posterior parietal cortex and the middle temporal cortex (18-21). It sends fibers to the SMA, the frontal eye field and the dorsal premotor cortex (20-22). Broca's motor area controls expressive speech and brings out the formation of words by connection with the primary motor area. Lesion of the area leads to motor aphasia characterized by the inability to express speech $(7,22)$.

The Prefrontal area (BA8, BA9, BA10, BA46, BA47) is located anterior to the motor and premotor areas. This area is related to the individual's personality and determines the judgment of the individual. It is related to the social and moral awareness of the person along with the emotional depth $(2,7,21,22)$. The anterior part of the para-hippocampal gyrus and uncus represents the olfactory area (BA28); the lesion in this area manifests as olfactory hallucination (2, 18, 22).

Primary sensory areas (BA3, BA1, BA2), also known as the primary somatosensory cortex, is located in the postcentral gyrus and extends into the posterior part of the paracentral lobule $(10,18,20)$. The BA3a is located in the depth of the central sulcus while the BA3b is located in the posterior wall of the central sulcus. $(2,10,20-22)$

The postcentral gyrus represents the opposite half of the body in an inverted manner; the face, lip and tongue are represented inferiorly while the trunk and upper limb are represented on the superolateral aspect (18-22). The area receives touch, pain, thermal sensations, and proprioceptive sensations from the opposite half of the body (18-22). Some areas like the pharynx, larynx and perineum have bilateral cortical representation (19-22). U shaped fibers connect the primary somatosensory area with the ipsilateral BA4, superior parietal lobule, inferior parietal lobule, and the parietal operculum (2, 5, 9, 18, 21-22).

The rostral area of the parietal operculum can be subdivided into OP1, OP2, OP3 and OP4. The secondary sensory area (Secondary somatosensory area, SII) is located at the upper lip of the posterior ramus of the lateral sulcus occupying 
OP1, OP3 and OP4 while OP2 represents the putative vestibular cortex. Although it receives all sensations, pain is dominantly represented in the area $(2,18,20,22)$.

The Superior parietal lobule represents the sensory association area (BA5, BA7) that is subdivided into 5L, 5M, 5Ci, 7A, 7P, 7M and 7PC. The secondary sensory area is related to stereognosis; it is concerned with the perception and recognition of the object by shape, size, texture, and roughness (18-22).

The posterior part of the superior temporal gyrus, angular gyrus (BA39) and supramarginal gyrus (BA40) of the left cerebral hemisphere (in $70 \%$ of subjects) represent Wernicke's sensory speech area. Wernicke's speech areas receive sensory input from the visual and auditory sensory area and interpret it. The angular and supramarginal gyri are concerned with the learning process through reading, writing, and computing. The lesion of the area leads to sensory aphasia with various characteristics, for example, inability to read (alexia), write (agraphia), compute (acalculia), and name objects (anomia). Lesion of Wernicke's speech area along with Broca's motor speech area leads to global aphasia characterized by the inability to produce speech, and understand written and spoken words $(2,5,18,20,22)$.

The lower part of the post-central gyrus and superior wall of the posterior sulcus of the lateral sulcus represents the taste (Gustatory) area that is located just posterior to the primary sensory area for the mouth (20-22).

Superior, middle, and inferior temporal gyri represent BA22, BA21 and BA20 respectively, while the temporal pole corresponds to the BA38 (2, 18-20). The primary auditory area (BA41) occupies the superior wall of the superior temporal gyrus (Heschl's gyrus/gyri) and is surrounded by the secondary auditory area (BA42) $(2,18)$. The primary auditory cortex is koniocortical in which layer III contains mainly small and medium-sized pyramidal cells arranged in radial columns which extend into the neighboring layers (creating 'Rain shower formation') (2, 18-20). The primary auditory area receives auditory sensations from the cochlea (Organ of Corti) via the medial geniculate body through the auditory radiation. It receives sensations based on loudness, quality, and sound of speech $(2,18,20)$.

The secondary auditory area (BA42) is situated posterior to the primary auditory area on the lateral surface of the superior temporal gyrus. It correlates the impulses received from the primary auditory area with the past auditory experiences. Lesion of the secondary auditory area leads to word deafness in which the patient fails to interpret the heard sound $(18,20)$.

Occipital lobe contains BA17, BA18 and BA19 (20-22). The primary visual area (V1; BA19) is the striate cortex located on the medial aspect of the occipital lobe occupying the upper lip, lower lip, and the depth of the calcarine sulcus (22). The visual area cortex also known as the striate area shows white stria of Gennari. It receives input from the lateral geniculate nucleus via the optic radiations $(5,19-22)$. The visual area receives optical fibers from the ipsilateral temporal half of the retina and contralateral nasal half of the retina; in addition, fibers from the superior retinal quadrant project into the superior wall of the post-calcarine sulcus and fibers from the inferior retinal quadrant project into the inferior wall of the post-calcarine sulcus $(5,20-22)$. The posterior one-third of the visual cortex represents the projection from the macular area of the retina that has the 
maximum visual acuity (Keenest vision) (5, 9, 20-22). Lesion of the primary visual area results in contralateral homonymous hemianopia characterized by the vision loss of the opposite half of the visual field $(5,20-22)$. The unilateral lesion of the inferior wall of post-calcarine sulcus results in superior quadrantic hemianopia and the lesion of the superior wall of the post-calcarine sulcus results in inferior quadrantic hemianopia $(5,9,20,22)$.

The visual area representing the macular part of the retina is spared in most cases of occipital cortical lesions; it leads to loss of peripheral vision with unaffected macular vision. The macular vision is spared because of the following reasons $(2,5,9,20)$ :

(i) The visual area representing the macular vision is extensive and separate from the area for the peripheral vision.

(ii) The macular area receives dual blood supply-from the posterior cerebral artery and the middle cerebral artery. In the case of the occipital lesion by thrombosis of the posterior cerebral artery, the macular area is spared.

The secondary visual area includes parastriate visual area II (BA18) and peristriate visual area III (BA19) that surrounds the primary visual area (BA17) $(18,20,22)$. The secondary visual area receives afferents from the primary visual area and has reciprocal connections with other cortical areas $(18,20,22)$. The projection fibers from the secondary visual area passing through the optic radiation connect the motor nuclei of extra-ocular muscles and the superior colliculus $(18,20,22)$. The secondary visual area helps in recognizing the object with the help of past experiences $(18,20,22)$. Secondary visual areas form an occipital eye field because stimulation of the areas produces conjugate deviation of the eyes to the opposite side $(18,20,22)$. This mechanism is a reflex mechanism unlike the voluntary mechanism of the frontal eye field (BA8) (9, 20-22).

Higher visual association area (BA39) is located in the angular gyrus of the inferior parietal lobule where further neural processing of visual impression takes place to comprehend the various objects and language symbols $(9,21)$. Lesion of the area leads to visual agnosia where the subject fails to recognize objects $(9,20,22)$.

\section{CEREBRAL DOMINANT HEMISPHERE}

Although the left and right cerebral hemispheres look alike in shape, size, and dimensions, they differ in their neuronal activities and functional areas $(2,18$, 21-23). Some established functional differences have been identified among the right and left cerebral hemispheres (21-23). The cerebral hemisphere controlling the language is considered as a dominant cerebral hemisphere $(18,21-23)$. In all right-handed people and almost half of the left-handed people, language is processed in the left hemisphere and therefore, the left hemisphere is the dominant hemisphere in $90-95 \%$ of the population (21-23). The dominant hemisphere is more proficient in handedness, language, mathematical and analytical processes (21-23). The opposite hemisphere is more proficient in creative arts, music, spatial comprehension, geometrical and facial recognition (21-23). 


\section{BLOOD SUPPLY OF THE CEREBRAL CORTEX}

The brain is supplied basically by four arteries: paired vertebral arteries and paired internal carotid arteries $(23,24)$. Two vertebral arteries unite with each other at the ventral surface of the pons to form the basilar artery; the basilar artery further divides to form the posterior cerebral arteries (24). The internal carotid artery divides into anterior and middle cerebral arteries (24). The anterior, middle, and posterior cerebral arteries communicate with each other and form an arterial circle known as the arterial circle of Willis $(23,24)$. The internal carotid artery enters the middle cranial fossa through the foramen lacerum by the side of the dorsum sellae (20-24). It passes first horizontally forward along the floor of the cavernous sinus, then ascends upward to the middle clinoid process, and courses backwards below the optic nerve $(2,5,22-24)$. It turns upward lateral to reach the anterior perforated substance and divides into anterior and middle cerebral arteries $(23,24)$. The directional changes of the internal carotid artery in the cranial cavity form a U-shaped bend known as a carotid siphon $(23,24)$. The cavernous part of the internal carotid artery gives superior and inferior hypophysial branches to the hypophysis cerebri (24). Other branches of the internal carotid artery are ophthalmic, posterior communicating, anterior choroid, anterior cerebral, and middle cerebral arteries $(23,24)$.

The anterior cerebral artery is directed medially above the optic nerve and then enters the median longitudinal fissure of the brain (24). It connects with the corresponding artery of the opposite side through the anterior communicating artery $(23,24)$. The anterior cerebral artery proceeds further back along the medial surface of the cerebral hemisphere in conformity with the corpus callosum curvature $(2,22-24)$. Then it turns upwards in front of the parieto-occipital sulcus $(23,24)$. The anterior cerebral artery gives cortical branches like an orbital branch, frontopolar branch, calloso-marginal branch and pericallosal branch except for the central medial striate branch (Recurrent artery of Heubner) $(23,24)$. The orbital branch supplies the orbital surface of the frontal lobe $(23,24)$. The frontopolar artery supplies the medial surface of the frontal lobe. The Callosomarginal artery supplies the cinguli gyrus and the paracentral lobule (24). The Pericallosal artery supplies the medial surface of the parietal lobe and pre-cuneus (22-24). Small branches from the anterior cerebral artery supply the lamina terminalis by two sets of capillary plexuses in the overlying pia mater which drains into the second sinusoidal plexuses (24). From the sinusoidal plexus, the blood drains into the hypothalamic veins; this portal system is a part of the 'Organusm vasculosum lamina termianlis' (24).

The middle cerebral artery is the large terminal branch of the internal carotid artery; it passes laterally along the stem of the lateral sulcus between the frontal and the temporal lobes. On the superolateral surface of the hemisphere, it runs in the posterior ramus of the lateral sulcus lying over the insular lobe (24). Here, it gives cortical branches to supply the insular lobe and lateral surface of the frontal, parietal, temporal, and occipital lobes (24). Branches of the middle cerebral artery are the lenticular-striate artery, the anterior temporal artery, the orbitofrontal artery, the pre-Rolandic and Rolandic arteries, the anterior parietal artery, the posterior parietal artery, posterior temporal artery, and angular artery (24). All branches of the middle cerebral artery are cortical except the lenticulo-striate 
branch (Charcot's artery of cerebral hemorrhage) (24). The middle cerebral artery supplies the lateral part of the orbital surface and superolateral surface of the cerebral hemisphere except a narrow strip near the superomedial border extending from the frontal pole of the cerebral hemisphere to the parieto-occipital sulcus (24). The middle cerebral artery supplies the sensory-motor areas around the central sulcus except for the area for the lower extremity, auditory area, motor speech area and sensory speech area $(18,20-24)$.

The posterior cerebral arteries are the paired terminal branches of the basilar artery. It joins with the posterior communicating artery to complete the circle of Willis then curves around the crus cerebri to reach the medial surface of the hemisphere beneath the splenium of the corpus callosum $(23,24)$. The posterior cerebral artery gives postero-lateral striate branch, posterior choroid artery, and cortical branches. The cortical branches give temporal and internal occipital branches $(23,24)$. The temporal branches supply the inferior surface of the temporal lobe (including the temporal and lingula gyri) except the temporal pole (24). The internal occipital artery further subdivides into parieto-occipital and calcarine branches that run along the parieto-occipital and calcarine sulcus, respectively $(23,24)$. Cuneus, pre-cuneus, and the strip adjoining the superolateral surface of the occipital pole is supplied by the branches of the internal occipital artery (24). The calcarine artery supplies the primary visual area; the occlusion of which may lead to the contralateral homonymous hemianopia (20-24). Posterolateral striate branches supply the pulvinar of the thalamus, geniculate bodies, and lateral thalamic nuclei $(23,24)$. The posterior choroid artery provides branches to the choroid plexus at the body of the lateral ventricle and third ventricle $(23,24)$.

\section{CONCLUSION}

Cerebral cortex controls the motor activities and reacts on the basis of sensations received through the thalamus. Understanding the anatomy of the cerebral cortex is crucial to identify the site of lesion in neurological pathologies.

Conflict of Interest: The authors declare no potential conflicts of interest with respect to research, authorship and/or publication of this manuscript.

Copyright and Permission Statement: The authors confirm that the materials included in this chapter do not violate copyright laws. Where relevant, appropriate permissions have been obtained from the original copyright holder(s), and all original sources have been appropriately acknowledged or referenced.

\section{REFERENCES}

1. Ekinci N, Acer N, Akkaya A, Sankur S, Kabadayi T, Sahin B. Volumetric evaluation of the relations among the cerebrum, cerebellum and brain stem in young subjects: a combination of stereology and magnetic resonance imaging. Surg Radiol Anat. 2008;30(6):489-494. https://doi.org/10.1007/ s00276-008-0356-z 
2. Bui T, M Das J. Neuroanatomy, Cerebral Hemisphere. In: StatPearls. Treasure Island (FL): StatPearls Publishing; 2021.

3. Uddin MA, Haq TU, Rafique MZ. Cerebral venous system anatomy. J Pak Med Assoc. 2006; 56(11):516-9.

4. Jin SW, Sim KB, Kim SD. Development and Growth of the Normal Cranial Vault : An Embryologic Review. J Korean Neurosurg Soc. 2016;59(3):192-6. https://doi.org/10.3340/jkns.2016.59.3.192

5. Ribas GC. The cerebral sulci and gyri. Neurosurg Focus. 2010;28(2):E2. https://doi.org/10.3171/ 2009.11.FOCUS09245

6. Nishikuni K, Ribas GC. Study of fetal and postnatal morphological development of the brain sulci. J Neurosurg Pediatr. 2013;11(1):1-11. https://doi.org/10.3171/2012.9.PEDS12122

7. Javed K, Reddy V, M Das J, Wroten M. Neuroanatomy, Wernicke Area. 2020 Jul 31. In: StatPearls. Treasure Island (FL): StatPearls Publishing; 2021 Jan-. PMID: 30422593.

8. Morriss-Kay GM, Wilkie AO. Growth of the normal skull vault and its alteration in craniosynostosis: insights from human genetics and experimental studies. J Anat. 2005 Nov;207(5):637-53. https:// doi.org/10.1111/j.1469-7580.2005.00475.x

9. Rehman A, Al Khalili Y. Neuroanatomy, Occipital Lobe. 2020 Jul 31. In: StatPearls. Treasure Island (FL): StatPearls Publishing; 2021 Jan-. PMID: 31335040.

10. Kier EL, Fulbright RK, Bronen RA. Limbic lobe embryology and anatomy: dissection and MR of the medial surface of the fetal cerebral hemisphere. AJNR Am J Neuroradiol. 1995 Oct;16(9):1847-53. PMID: 8693985.

11. Tamraz JC, Comair YG: Atlas of Regional Anatomy of the Brain Using MRI. Berlin: Springer; 2000. $325 \mathrm{p}$.

12. Squire LR, Bloom FE, McConnell SK, Roberts JL, Spitzer NC, Zigmond MJ: Fundamental Neuroscience, 2nd ed. Amsterdam: Academic Press, 2003

13. GarciaL P, Garcia M, Freire M. The histological slides and drawings of Cajal. Front. Neuroanat;2010:4-9.

14. García-CM, Hacker JL, Zikopoulos B. A Protocol for Cortical Type Analysis of the Human Neocortex Applied on Histological Samples, the Atlas of Von Economo and Koskinas, and Magnetic Resonance Imaging. Front. Neuroanat. 2020: 14:576015. https://doi.org/10.3389/fnana.2020.576015

15. Ross MH, Pawlina W. Histology: A Text and Atlas: with Correlated Cell and Molecular Biology. 8th ed. Baltimore, MD: Lippincott Wiliams \& Wilkins; 2018. 1045 p.

16. Amunts K, Zilles K. Architectonic Mapping of the Human Brain beyond Brodmann.Neuron. 2015;88(6):1086-1107. Douglas RJ, Martin KA. Neuroanl circuits of the neocortex. Annu. Rev. Neurosci.2004;27:419-451. https://doi.org/10.1016/j.neuron.2015.12.001

17. Broca P. Anatomie comparée des circonvolutions cérébrales. Le grand lobe limbique et la scissure limbique dans la série des mammifères. Rev Anthrop 1978; 2: 285-498.

18. El-Baba RM, Schury MP. Neuroanatomy, Frontal Cortex. 2020 Jul 31. In: StatPearls .Treasure Island (FL): StatPearls Publishing; 2021.

19. Catani M. The clinical anatomy of the temporal and parietal lobes. Cortex. 2017;97:160-163. https:// doi.org/10.1016/j.cortex.2017.11.006

20. Vernet M, Quentin R, Chanes L, Mitsumasu A, Valero-Cabré A. Frontal eye field, where art thou? Anatomy, function, and non-invasive manipulation of frontal regions involved in eye movements and associated cognitive operations. Front Integr Neurosci. 2014;8:66. https://doi.org/10.3389/fnint. 2014.00088

21. Flinker A, Korzeniewska A, Shestyuk AY. Redefining the role of Broca's area in speech. Proc Natl Acad Sci U S A. 2015;112(9):2871-2875. https://doi.org/10.1073/pnas.1414491112

22. Corballis MC. Left brain, right brain: facts and fantasies. PLoS Biol. 2014;12(1):el001767. https://doi. org/10.1371/journal.pbio.1001767

23. Burlakoti A, Kumaratilake J, Taylor J, Henneberg M. Asymmetries of total arterial supply of cerebral hemispheres do not exist. Heliyon. 2019;5(1):e01086. https://doi.org/10.1016/j.heliyon.2018. e01086

24. Avci E, Fossett D, Aslan M, Attar A, Egemen N. Branches of the anterior cerebral artery near the anterior communicating artery complex: an anatomic study and surgical perspective. Neurol Med Chir (Tokyo). 2003;43(7):329-333. https://doi.org/10.2176/nmc.43.329 